Service as it is now. We also discovered that it might be possible to identify the likely demand on psychiatric services from sociodemographic factors and that useful comparisons could be made between Districts in terms of the amount of total activity (energy spent or work done) of the service in comparison to the resources they have, and in comparison to the potential demand.

If there is very little going in the way of out-patients' admissions, DVs, day hospital visits, CPN visits, but there is a large resource then one may suspect that something is wrong and local managers can at least try to explain it. There may be perfectly good explanations, for example the work may be going into some other area of activity like psychotherapy and rehabilitation which is not being measured. Equally, if there are very low resources or very low activity in respect to a high potential demand, as we found in some Districts, then it points to the fact that the District has not adequately resourced their mental health service.

Dr Thomas' points are important ones, but the measures we have suggested are perhaps analogous to the IQ-it measures one aspect of functioning and it has useful purposes but it does not give an indication of the quality of the individual nor their likely success in work and examinations later in life. When one aspect of the IQ is discrepant with another it can indicate that something is wrong and needs to be examined more closely. One should not have to repeat that any type of statistics have to be used intelligently and for the purposes to which they are suited. Dr Thomas's worry is that our approach might be used to estimate the quality of the service or other factors which, of course, is not the intention.

There are real problems with the kind of algorithm we used and by no means do I think the weightings we have given to different aspects of the service are right. I think we were trying to achieve, in measuring activities, exactly what Dr Thomas says-a measure of the work done or energy expended, rather than the quality or usefulness of the work done. The latter has to do with efficacy and, as I stated in my paper, we did not attend to that particular issue. We also recognised that as the nature of psychiatric services changes and the shift of emphasis moves from the hospital to the community, it will be necessary to add new ways of measuring both the work done and its efficacy. In the meantime, the work around a psychiatric admission is much, much greater than the work which surrounds an individual CPN visit and the number of out-patient visits as a total represent a far greater amount of work than the amount of work done by a small number of CPNs in most catchment areas.

I would hope that our report will move others to take up these issues and try to develop better measures of efficacy as well as efficiency-indeed we are currently applying for grants which begin in this direction.

The points made in Dr Thomas' letter need to be said and I hope that he and others will begin to work in the directions that he has outlined.

Charing Cross Hospital, London W6

\section{Training in psychotherapy}

\section{Dear Sirs}

Despite the College's many requirements for higher psychotherapy training, it could be argued that key elements are lacking without which no specialist training in therapy can be said to be adequate. This letter hopes to stimulate debate about these hiati. Some might consider it a counsel of perfection, but the ideas could in fact be built into many training programmes within a short time, given the will to do so. Among the main training requirements missing at present are:

(1) Systematic training, academic and clinical, in the indications for each type of psychotherapy and why each patient of trainees is selected for a given approach. Where possible this training should be in the light of the research evidence on efficacy and, where that is lacking, of welldetailed clinical experience. The therapeutic investment in trainees' patients may range from 10 to fully 250 sessions, yet trainees are insufficiently schooled in how to decide whether therapy should be long rather than brief, family or group rather than individual, dynamic rather than behavioural, etc. There are of course huge gaps in our knowledge about some of these issues but much is known that is not taught. Trainees' time is a precious but limited resource which they need to learn to deploy wisely; one patient having 250 sessions denies 25 other patients having 10 sessions each, a decision that is worthwhile at times but should be clearly justified.

(2) Systematic training, academic and clinical, in defining with most patients the goals of therapy at the outset and the criteria by which to judge their subsequent attainment, in rapidly measuring goal and criteria attainment at the start, at intervals during therapy, and at follow-up, and in relating such change to their clinical interventions. Trainees give this too little attention. The success of psychotherapy training in teaching trainees to help their patients cannot be adequately judged without ascertaining clinical change. The means to do this economically on a routine, not research, basis has been available for decades even in dynamic psychotherapy at the Tavistock.

(3) Follow-up of each patient for at least six months where possible to check the durability of changes ensuing from therapy. If properly planned for, follow-up should be feasible with most patients in a four-year training programme, but at present it is given little attention.

(4) Systematic academic training in the epidemiological base relevant to psychotherapy. At present trainees have a little knowledge gleaned from general psychiatry yet on becoming consultants will be expected to help plan psychotherapy services. Informed judgement requires sound epidemiological knowledge, much of which is available despite a lack of data on some key issues. The therapeutic emphasis on trainees' therapy for the minority of sufferers who attend hospital reflects insufficient time given to the bulk of problems amenable to brief psychotherapy which remain in primary care. Another instance of inattention to what is common and treatable is a general lack of experience in 
helping sexual disorders, a deficiency easily remediable in a four-year training programme.

A different point reflects some of the above concerns. Many trainers think that personal therapy for psychotherapy trainees is central to training. This greatly increases training cost, narrowing the time available for other psychotherapy practice, study and research. Expensive recommendations like that for personal training would be better based on research results than on opinion. It would be timely to compare the outcome of patients treated by psychiatrists with and without personal training to see whether such a costly procedure is justified from the patient's point of view, or whether it should rather be made optional.

Some might feel that the chief guide to higher training should be trainees' preference for whatever forms of psychotherapy and patient problem happen to interest them, however limited those may be. Would we accept a general psychiatrist's argument that he wished to learn about and prescribe only a handful of drugs for a minority of patients in the population he served, as he was bored by the many other effective ones for the rest? The varied problems in the population tended by consultant psychotherapists would gain more from psychotherapists able to apply and supervise most of the methods likely to help those problems, especially the common ones, than from superspecialists schooled only in approaches useful for a small minority of cases. A whole four years of specialist training allows for diversification.

Institute of Psychiatry

ISAAC MARKS

London SES

\section{Benzodiazepines and dependence \\ DeAr SiRS}

I am astonished to read this report in the March issue of the Bulletin. At a time when the public and the profession have finally got the message that these drugs should not be prescribed, the College publishes a statement explaining how they should be prescribed. Who are these patients with anxiety which is disabling, severe or subjecting the individual to unacceptable distress and who require short-term relief? Similarly, to which patients with insomnia does the statement refer? I would challenge the Committee to publish half a dozen vignettes of such patients in say five or six lines each as guidance to members of the College of what they mean. We might then be in a position to know what we are really talking about.

I cannot remember the last time I prescribed a benzodiazepine (except occasionally for the control of extremely disturbed psychotic behaviour in in-patients) and I have not felt the lack of them in treating a large number of patients with anxiety symptoms. Patients sometimes ask for tablets at the beginning of a consultation; after careful enquiry into the sources of anxiety and a discussion of how these might be remedied, a request is not usually repeated. Professor Anthony Clare has written of his concern for the "medicali- sation of what are seen to be social problems" and refers to these drugs being "potentially hazardous".' Professor W. H. Trethowan coined the phrase "pills for personal problems" as prophylaxis against the irrationality of offering help in chemical terms where more rational, that is more scientific methods of intervention, would be appropriate. I have already written ${ }^{2}$ that the time has arrived to state clearly that there is no use for these drugs in the treatment of anxiety and have referred to their role in generating symptoms including insomnia.

The report does not refer to the dangers of benzodiazepine drugs in the elderly and I would refer readers to the Prescribers Journal of December 1987 where Professor Elaine Murphy lists benzodiazepines under the heading 'Drugs to avoid'.

In conclusion I refer to the paragraph headed Depression. This states that depression is not an indication, it then goes on to say that the drugs may be prescribed under certain conditions and finally says how dangerous they are in that they may precipitate suicide. What is the College recommending? The paragraph then goes on to say that withdrawal may precipitate depression. Having withdrawn these drugs from large numbers of patients I must say that I have never seen anything but benefit although, of course, if benzodiazepines are used where there has been a failure to identify symptoms as having a depressive basis these symptoms may appear in greater force when the drugs are stopped. Patients who have been taking these drugs for a substantial period of time are sometimes angry if one suggests that they should stop them and their upset state is not uncommonly miscontrued as depression. If patients are told that stopping the tablets might ultimately improve their sleep and reduce their level of anxiety and if this is done sympathetically and with suitable explanation of what to expect, such patients frequently become amongst the most appreciative patients a psychiatrist can have.

The London Hospital Medical

SAMUEl I. COHEN

College, London EI

\section{REFERENCES}

${ }^{1}$ A. Clare (1987) The Benzodiazepines in Current Clinical Practice. (eds H. Freeman and Y. Rue) Royal Society of Medicine International Congress and Symposium Series, 114.

${ }^{2}$ CoHEN, S.I. (1987) Are benzodiazepines useful in anxiety? The Lancet, ii, 1080.

\section{DeAR Sirs}

The College Statement: Benzodiazepines and Dependence (Bulletin, March 1988, 12, 107-109) commences with indications for the prescription of these drugs. Sections l(a), (b) and (i) refer respectively to the use of benzodiazepines in 'anxiety', 'insomnia' and 'depression' accompanied by anxiety'; another section refers to 'excitement, agitation and severe psychotic disturbance'. In the first three of these 'indications' it is recommended that these drugs should be prescribed only when the condition is "disabling, severe and causing extreme distress"; in the first and third 'indication' 